\title{
Hydrogen-vacancy related defect in chemical vapor deposition homoepitaxial diamond films studied by electron paramagnetic resonance and cathodoluminescence
}

\author{
N. Mizuochi ${ }^{\mathrm{a})}$ \\ Diamond Research Center, AIST, Tsukuba Central 2, Tsukuba, 305-8568, Japan, \\ Graduate School of Library, Information and Media Studies, University of Tsukuba, \\ Tsukuba, 305-8550, Japan, and CREST JST, Chiyoda Tokyo, 102-0081, Japan \\ H. Watanabe, H. Okushi, and S. Yamasaki \\ Diamond Research Center, AIST, Tsukuba Central 2, Tsukuba, 305-8568, Japan \\ and CREST JST, Chiyoda Tokyo, 102-0081, Japan \\ J. Niitsuma and T. Sekiguchi \\ National Institute for Materials Science, Tsukuba, 305-0044, Japan
}

(Received 29 July 2005; accepted 4 January 2006; published online 2 March 2006)

\begin{abstract}
Hydrogen-vacancy related defect $\left(H 1^{\prime}\right)$ in chemical vapor deposition homoepitaxial diamond films has been investigated by electron paramagnetic resonance and cathodoluminescence. It is found that the concentration of $\mathrm{H}^{\prime}$ ' significantly decreases as the dilution $\left(\mathrm{CH}_{4} / \mathrm{H}_{2}\right)$ ratio decreases. It is also confirmed that the intensity of free-exciton emission $\left(I_{\text {ex }}\right)$ increases as the $\mathrm{CH}_{4} / \mathrm{H}_{2}$ ratio decreases. The complementary relationship between $I_{\mathrm{ex}}$ and $H 1^{\prime}$ can be explained by considering that $H 1^{\prime}$ acts as a nonradiative recombination center which reduces the lifetime of free exciton and $I_{\mathrm{ex}}$. The suppression mechanism of $H 1^{\prime}$ is discussed by considering the balance between the growth rate and the annihilation rate of $\mathrm{H1}^{\prime}$ in the subsurface region. (c) 2006 American Institute of Physics.
\end{abstract} [DOI: $10.1063 / 1.2176860]$

Diamond is a promising material of optical devices for ultraviolet light emission and electronic devices for high power, high frequency, etc. because of its superior physical and electrical properties. ${ }^{1,2}$ As a result of the development of chemical vapor deposition (CVD) growth techniques, high quality diamond films with high Hall mobilities ${ }^{2-4}$ and an atomically flat surface have been synthesized. ${ }^{2}$

For ultraviolet light emitting diodes, free-exciton (FE) emission $(235 \mathrm{~nm})$ is considered to be a candidate for a light source. From cathodoluminescence (CL) studies, it was reported that the intensity of FE emission $\left(I_{\mathrm{ex}}\right)$ significantly increases as the dilution $\left(\mathrm{CH}_{4} / \mathrm{H}_{2}\right)$ ratio decreases in homoepitaxial CVD diamond film growth., ${ }^{2,5}$ It is considered that the increase of $I_{\mathrm{ex}}$ results from the suppression of defects or impurities in the film grown with the extremely low $\mathrm{CH}_{4} / \mathrm{H}_{2}$ ratio of $0.025 \%$. However, there has been no quantitative study on the suppression of defects.

In CVD polycrystalline ${ }^{6}$ and CVD monocrystalline $e^{7,8}$ diamond, carbon dangling bond $(\mathrm{C} \mathrm{db})$ defects accompanying a hydrogen atom $(\mathrm{H})$ such as $H 1, H 2$, and $V H^{-}$have been reported. It has been suggested that $H 1$ is a neutral charge state of $\mathrm{H}$-vacancy defects ${ }^{6,7}$ and that $H 2$ is similar to $H 1$ but the distance between $\mathrm{C} \mathrm{db}$ and $\mathrm{H}$ is longer than that of $H 1{ }^{6}$ It has also been suggested that $V H^{-}$is the negative charge state of a H-vacancy complex. ${ }^{8}$ Recently, we observed $\mathrm{C} \mathrm{db}$ defects accompanying $\mathrm{H}$ in high quality undoped and boron (B) doped $p$-type CVD homoepitaxial diamond films by electron paramagnetic resonance (EPR), ${ }^{9,10}$ which we tentatively call $H 1^{\prime}$ in this letter. The nearby $\mathrm{H}$ was confirmed by the EPR spectrum with shoulders. The spectrum of $H 1^{\prime}$ cannot be reproduced by simulation using the parameters of $H 1$, $H 2$, and $V H^{-}$centers. ${ }^{10}$ Recently, it was also reported by elec-

${ }^{a)}$ Electronic mail: mizuochi@slis.tsukuba.ac.jp tron nuclear double resonance experiments that the distance between $\mathrm{H}$ and $\mathrm{C} \mathrm{db}$ in H-related defects in a P-doped CVD diamond film in (111) substrate is not as close as that for $H 1{ }^{11}$ These results suggest that $H 1^{\prime}$ originates from the combination of a couple of $\mathrm{H}$-vacancy complexes with different distance between $\mathrm{H}$ and $\mathrm{C} \mathrm{db}$, although more intensive research is needed to obtain a conclusive structural model.

In our growth condition of $\mathrm{CH}_{4} / \mathrm{H}_{2}=0.3 \%-0.5 \%, \mathrm{H}^{\prime}$ is most likely a dominant defect which has the largest concentration $\left.\left(\sim 10^{18} / \mathrm{cm}^{3}\right)\right)^{9,10}$ It was shown that $H 1^{\prime}$ is distributed uniformly along the depth direction, indicating bulk defects. ${ }^{9}$ It is stable even after annealing at $1573 \mathrm{~K} .{ }^{9,10}$ Therefore, it is important to reveal its effects on optical and electrical properties. In this study we clarified the relationship between $I_{\mathrm{ex}}$ and $H 1^{\prime}$, and discuss the role of it on $I_{\mathrm{ex}}$.

CVD diamond films were deposited epitaxially on HPHT IIa diamond (001) substrates with dimensions of 2.5 $\times 2.5 \times 0.5 \mathrm{~mm}^{3}$. The misorientation angles $\left(\theta_{\text {off }}\right)$ of the substrate are in the rangeof 3-7 deg. The films were grown by a microwave (MW) plasma-enhanced CVD reactor using $\mathrm{CH}_{4}$ diluted with $\mathrm{H}_{2}$ at $1073 \mathrm{~K}$. The total gas pressure, total gas flow rate, and input MW power were maintained constant at 25 Torr, $400 \mathrm{sccm}$, and $750 \mathrm{~W}$, respectively. Deposition durations, $\mathrm{CH}_{4} / \mathrm{H}_{2}$ ratios, and thicknesses of films were summarized in Table I. The thickness was estimated by the step height between the region with and without a diamond mask on the substrates. The details of EPR measurements are described elsewhere. ${ }^{9,10}$ The magnetic field was calibrated by simultaneously measuring neutral nitrogen $(\mathrm{N})$ at a substitutional position $(\mathrm{P} 1)^{12}$ in the substrate. The CL measurements were performed at $100 \mathrm{~K}$ and acceleration voltage of $10 \mathrm{keV}$, which corresponds to a penetration depth of about $1 \mu \mathrm{m} .{ }^{13}$ The details of $\mathrm{CL}$ spectrometers are described elsewhere. ${ }^{5,14}$ 
TABLE I. The growth condition and concentration of $H 1^{\prime}\left(C_{H 1^{\prime}}\right)$.

\begin{tabular}{lcrcc}
\hline \hline Film & $\mathrm{CH}_{4} / \mathrm{H}_{2} \%$ & $T / h^{\mathrm{a}}$ & Thickness $/ \mu \mathrm{m}$ & $C_{H 1^{\prime}} / \mathrm{cm}^{3}$ \\
\hline I & 0.5 & 6 & 2.0 & $(3 \pm 1) \times 10^{18}$ \\
II & 0.5 & 6 & 1.8 & $(5 \pm 2) \times 10^{18}$ \\
III & 0.15 & 29 & 3.0 & $(1 \pm 0.4) \times 10^{18}$ \\
IV & 0.1 & 29 & 2.0 & $(5 \pm 2) \times 10^{17}$ \\
V & 0.035 & 200 & 2.7 & $(7 \pm 3) \times 10^{16}$ \\
\hline \hline
\end{tabular}

${ }^{\mathrm{a}} T$ is deposition duration.

In secondary ion mass spectroscopy, unintentionally incorporated $\mathrm{N}$ and $\mathrm{B}$ were not detected in our films, which means below about $2 \times 10^{17} / \mathrm{cm}^{3}$ for $\mathrm{N}$ and about 5 $\times 10^{15} / \mathrm{cm}^{3}$ for B. From EPR, no signal other than $H 1^{\prime}$ was detected in spite of measurements over a wide range of temperature and MW power. Only $P 1$ centers in the IIa substrates were observed and their intensity did not change after the film deposition.

Figure 1 shows the EPR spectra of $H 1^{\prime}$. The $g$ value was estimated to be $2.0026 \pm 0.0001$. The observed EPR line shape with a pair of partly resolved satellite lines with the splitting of $1.25 \mathrm{mT}$ corresponds to that of $H 1^{\prime}{ }^{9,10}$ These facts indicate that the defects observed in all films in this study are mainly $H 1^{\prime}$ because of the similarity of all spectra. In Fig. 2(a) and Table I, the concentration of $H 1^{\prime}\left(C_{H 1^{\prime}}\right)$ with respect to $\mathrm{CH}_{4} / \mathrm{H}_{2}$ ratio is represented. As can be seen, $C_{H 1^{\prime}}$ significantly decreases as the $\mathrm{CH}_{4} / \mathrm{H}_{2}$ ratio decreases.

As shown in Table $\mathrm{I}$, the growth rate also decreases as the $\mathrm{CH}_{4} / \mathrm{H}_{2}$ ratio decreases due to a balance of carbon species supplied and the effect of $\mathrm{H}$ etching. ${ }^{15}$ Figure 2(b) shows the relationship between $C_{H 1}$, and growth rate; $C_{H 1}$, is almost linearly proportional to the growth rate, indicating that the suppression of $H 1^{\prime}$ is realized by the slow growth rate.

Figure 3 shows the CL spectrum of the film III. The strong emissions were attributed to FE emission associated with a transverse optical phonon $(235 \mathrm{~nm})$ and its replica $(242 \mathrm{~nm})$, a transverse acoustic phonon $(233 \mathrm{~nm})$, and a longitudinal optical phonon $(238 \mathrm{~nm}){ }^{5,16}$ At $420 \mathrm{~nm}$, a very weak band-A emission was observed. ${ }^{17}$ In Fig. 4 , the intensity at $\lambda=235 \mathrm{~nm}\left(I_{\mathrm{ex}}\right)$ is represented as a function of $\mathrm{CH}_{4} / \mathrm{H}_{2}$ ratio and $C_{H 1^{\prime}}$, showing that $I_{\mathrm{ex}}$ increases with decreasing $\mathrm{CH}_{4} / \mathrm{H}_{2}$ ratio and $C_{H 1^{\prime}}$. This complementary relationship between $I_{\mathrm{ex}}$ and $C_{H 1^{\prime}}$ suggests that $H 1^{\prime}$ acts as a recombination center for FE. In addition, we did not observe radiative centers related to $H 1^{\prime}$, suggesting that the recombination takes place by nonradiative processes.

In general, $I_{\mathrm{ex}}$ is proportional to the internal quantum efficiency $\eta$, which is given by

$$
\eta=\frac{1 / \tau_{R}}{\left(1 / \tau_{R}\right)+\sum\left(1 / \tau_{\mathrm{NR}}\right)}
$$

where $\tau_{R}$ and $\tau_{\mathrm{NR}}$ are the lifetimes of radiative and nonradiative recombination, respectively. ${ }^{18}$ It is known that $\tau_{\mathrm{NR}}$ is inversely proportional to the concentration of nonradiative recombination center $(N)$, the capture cross section of recombination center $(\sigma)$, and the thermal velocity of carrier $(\nu)$ : $\tau_{\mathrm{NR}}^{-1}=\sigma \nu N{ }^{1,18}$ In the case that $H 1^{\prime}$ acts as one of the nonradiative recombination centers, Eq. (1) can be rewritten as Downloaded 02 Mar 2006 to 150.29.104.223. Redistribution subje

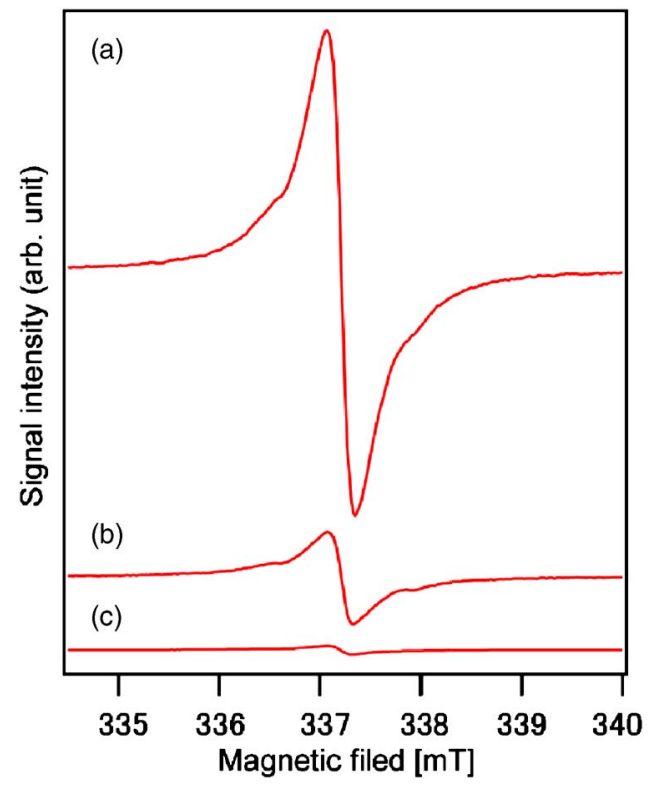

FIG. 1. The EPR spectra of $H 1^{\prime}$ in the films of (a) I, (b) III, and (c) V, respectively, at $40 \mathrm{~K}$. The MW frequencies are $9.45 \mathrm{GHz}$. The MW power and the amplitude of $100 \mathrm{kHz}$ field modulation were $0.1 \mathrm{~mW}$ and $0.07 \mathrm{mT}$, respectively. The signal intensities are normalized to compare their relative intensities.

$$
\eta=\frac{1 / \tau_{R}}{\left(1 / \tau_{R}\right)+\sum\left(1 / \tau_{\mathrm{NR}}^{\prime}\right)+\sigma_{H 1^{\prime}} \nu C_{H 1^{\prime}}}=\frac{c_{1}}{c_{1}+c_{2}+C_{H 1^{\prime}}},
$$

where $c_{1}=\left(\tau_{R} \sigma_{H 1^{\prime}} \mathrm{v}\right)^{-1}, c_{2}=\Sigma\left(\tau_{N R}^{\prime} \sigma_{H 1^{\prime}} \mathrm{v}\right)^{-1}$, and $\sigma_{H 1^{\prime}}$ is $\sigma$ of $H 1^{\prime}$. Previously, scanning CL measurements revealed that $I_{\mathrm{ex}}$ complementarily relates to band- $A$ emission mainly originating from nonepitaxial crystallites (NC). ${ }^{17} \mathrm{NC}$ and band- $A$ emission of the films in this study were not suppressed completely as shown in Fig. 3 and were almost independent of the $\mathrm{CH}_{4} / \mathrm{H}_{2}$ ratio. Therefore, $\mathrm{NC}$ is considered to be the recombination center which mainly determines $c_{2}$ in our films. Because the density and the largeness of NC are almost the same among the films of the present study, $c_{2}$ was assumed to be constant in our preliminary analysis. As shown in Fig. 4(b), the relationship between $C_{H 1^{\prime}}$ and $I_{\text {ex }}$ is well fitted by Eq. (2), which supports that $H 1^{\prime}$ acts as a nonradiative recombination center. From the least squares fitting, $\left(c_{1}+c_{2}\right)$ is estimated to be $5 \times 10^{17} / \mathrm{cm}^{3}$.

From optical studies, $\tau_{R}$ is reported to be in the range of $2.5 \times 10^{-7}-2.3 \times 10^{-6} \mathrm{~s}^{19,20}$ The typical value of $\sigma$ is in the range of $10^{-15}-10^{-17} \mathrm{~cm}^{2},{ }^{1,21}$ and that of $\nu$ is in the range of $10^{6}-10^{7} \mathrm{~cm} / \mathrm{s}$. From these estimations, $\left(\tau_{R} \sigma_{H 1}, v\right)^{-1}\left(=c_{1}\right)$ is calculated to be roughly in the range of $10^{14}-10^{18} / \mathrm{cm}^{3}$. The estimated value of $\left(c_{1}+c_{2}\right)$ is also in this range. Previously, it was reported that an atomically flat surface was realized resulting in significant suppression of $\mathrm{NC}$ and band- $A$ emission in the film grown with the extremely low $\mathrm{CH}_{4} / \mathrm{H}_{2}$ ratio of $0.025 \%$ on the substrate with $\theta_{\text {off }}$ of less than $0.5 \mathrm{deg}$ of $\mathrm{Ib}$ substrates. ${ }^{2,5}$ This film showed quite strong $I_{\mathrm{ex}}$ of more than 80 times that of the film grown with $\mathrm{CH}_{4} / \mathrm{H}_{2}=0.5 \%$. ${ }^{2} \mathrm{Al}-$ though $C_{H 1^{\prime}}$ could not be estimated in this film due to the overlap of the strong $P 1$ EPR signals in Ib substrates, the strong $I_{\mathrm{ex}}$ was beyond the expected value of Eq. (2) at $C_{H 1^{\prime}}=0$ when $c_{1}=5 \times 10^{17} / \mathrm{cm}^{3}$ and $c_{2}=0$. In order to understand the strong $I_{\mathrm{ex}}$ in the previous study, $c_{2}$ in the films of the present study should be in the range of $4-5 \times 10^{17} / \mathrm{cm}^{3}$
to AIP license or copyright, see http://apl.aip.org/apl/copyright.jsp 

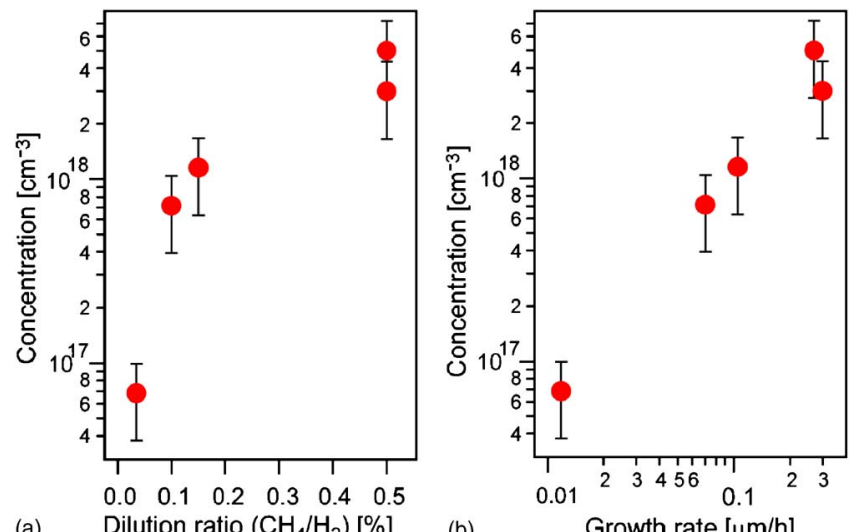

FIG. 2. The concentration of $H 1^{\prime}$ with respect to (a) the $\mathrm{CH}_{4} / \mathrm{H}_{2}$ ratios and (b) the growth rates.

and $c_{2}$ in the previous study can be considered to be less than $1 \times 10^{17} / \mathrm{cm}^{3}$ due to significant suppression of NC.

Next, the mechanism of the growth rate dependence on $C_{H 1^{\prime}}$ is discussed. We reported that $H 1^{\prime}$ is stable even after annealing at $1573 \mathrm{~K}$ which is much higher than the growth temperature $(1073 \mathrm{~K}){ }^{9,10}$ Therefore, $H 1^{\prime}$ created at certain layers from the top surface (subsurface region) during deposition remains. It is reasonable that within the subsurface region, $H 1^{\prime}$ can be annihilated because of high structural freedom at the surface or reaction with supplied carbon species. It can be considered that the number of $H 1^{\prime}$ at the subsurface region is determined by the ratio between annihilation time and the time existing in the subsurface region. The time existing in the subsurface region is inversely proportional to the growth rate. Under this mechanism, the suppression of $C_{H 1}$, due to the slow growth is expected to be enhanced in comparison with the fast growth.

We observed $\mathrm{C}$ db defects by EPR in N-doped CVD diamond film ${ }^{22}$ grown with higher MW power and more than 100 times higher growth rate compared with the film II. The order of concentration was almost the same with the film II. Although they cannot be straightforwardly compared due to different growth conditions, it is considered that there may be saturating mechanism of defect creation in it.

In summary, from the dependence of $\mathrm{CH}_{4} / \mathrm{H}_{2}$ ratio in CVD homoepitaxial diamond films, EPR revealed significant suppression of $H 1^{\prime}$ as the $\mathrm{CH}_{4} / \mathrm{H}_{2}$ ratio decreases. The mechanism of it was suggested by considering the balance

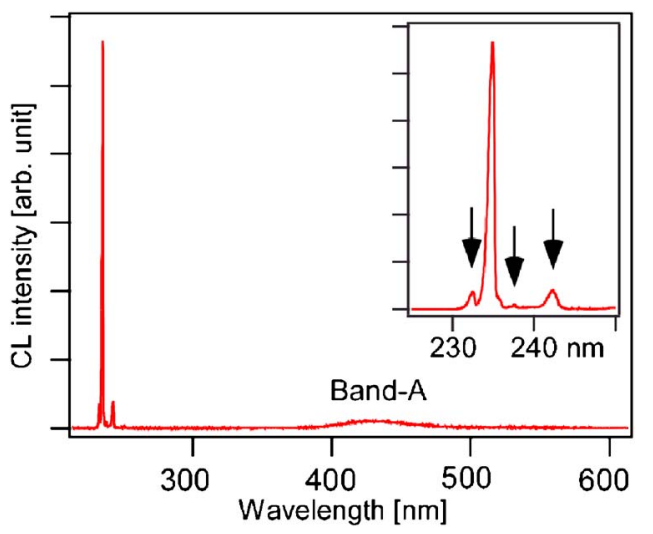

FIG. 3. The CL spectra of film III at $100 \mathrm{~K}$ with high resolution condition. The acceleration voltages of $10 \mathrm{keV}$. The inserted figure is the expanded CL spectrum in the UV region.
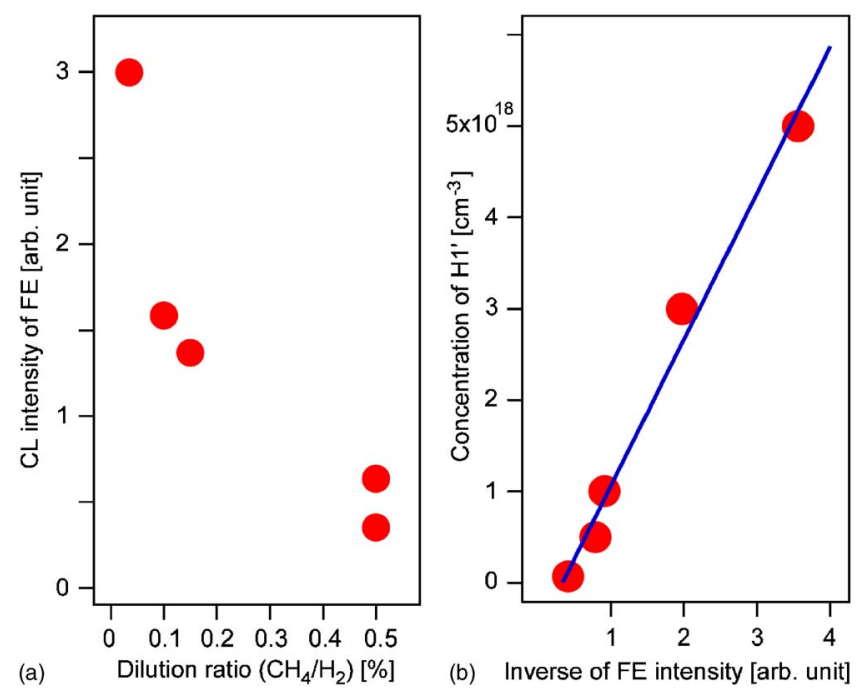

FIG. 4. The FE signal intensity with respect to $\mathrm{CH}_{4} / \mathrm{H}_{2}$ ratio. (b) The concentration of $H 1^{\prime}$ with respect to the FE signal intensity. The solid line in (b) is the least squares fitting curve by using Eq. (2).

between the growth rate and the annihilation rate. The complementary relationship between $I_{\mathrm{ex}}$ and $H 1^{\prime}$ was obtained by CL and EPR, which suggests that $H 1^{\prime}$ acts as a nonradiative recombination center which reduces $I_{\mathrm{ex}}$.

This work was partly supported by NEDO. The authors thank Dr. C. Nebel, Dr. A. Chayahara, and Y. Mokuno in AIST for helpful discussions and the supply of the high growth rate sample.

${ }^{1}$ D. L. Dreifus, in Diamond: Electronic Properties and Applications, edited by L. S. Pan (Kluwer Academic, Dordrecht 1995), Chap. 10, pp. 371-423; S. Grot, in ibid.443-457.

${ }^{2}$ H. Okushi, Diamond Relat. Mater. 10, 281 (2001).

${ }^{3}$ M. Katagiri, J. Isoya, S. Koizumi, and H. Kanda, Appl. Phys. Lett. 85, 6365 (2004).

${ }^{4}$ H. Kato, H. Okushi, and S. Yamasaki, Appl. Phys. Lett. 86, 222111 (2005).

${ }^{5}$ H. Watanabe and H. Okushi, Jpn. J. Appl. Phys., Part 2 39, L835 (2000).

${ }^{6}$ X. Zhou, G. D. Watkins, K. M. McNamara Rutledge, R. P. Messmer, and S. Chawla, Phys. Rev. B 54, 7881 (1996).

${ }^{7}$ K. Iakoubovskii, A. Stesmans, K. Suzuki, A. Sawabe, and T. Yamada, Phys. Rev. B 66, 113203 (2002).

${ }^{8}$ C. Glover, M. E. Newton, P. M. Martineau, S. Quinn, and D. J. Twitchen, Phys. Rev. Lett. 92, 135502 (2004).

${ }^{9}$ N. Mizuochi, H. Watanabe, J. Isoya, H. Okushi, and S. Yamasaki, Diamond Relat. Mater. 13, 765 (2004).

${ }^{10}$ N. Mizuochi, M. Ogura, H. Watanabe, J. Isoya, H. Okushi, and S. Yamasaki, Diamond Relat. Mater. 13, 2096 (2004).

${ }^{11}$ M. Katagiri, J. Isoya, S. Koizumi, and H. Kanda, Phys. Status Solidi A 201, 2451 (2004).

${ }^{12}$ K. Iakoubovskii and A. Stesmans, Phys. Status Solidi A 186, 199 (2001).

${ }^{13}$ K. Kanaya and S. Okayama, J. Phys. D 5, 43 (1972).

${ }^{14}$ T. Sekiguchi and K. Sumino, Rev. Sci. Instrum. 66, 4277 (1995).

${ }^{15}$ S. Ri, H. Yoshida, S. Yamanaka, H. Watanabe, D. Takeuchi, and H. Okushi, J. Cryst. Growth 235, 300 (2002).

${ }^{16}$ P. J. Dean, E. C. Lightowlers, and D. R. Wight, Phys. Rev. 140, A352 (1965).

${ }^{17}$ D. Takeuchi, H. Watanabe, S. Yamanaka, H. Okushi, H. Sawada, H. Ichinose, and T. Sekiguchi, Phys. Rev. B 63, 245328 (2001).

${ }^{18}$ J. I. Pankove, Optical Processes in Semiconductors (Prentice-Hall, Englewood Cliffs, NJ, 1975), p. 166.

${ }^{19}$ A. Fujii, K. Takiyama, R. Maki, and T. Fujita, J. Lumin. 94, 355 (2001).

${ }^{20}$ K. Takiyama, M. I. Abd-Elrahman, T. Fujita, and T. Oda, Solid State Commun. 99, 793 (1996).

${ }^{21}$ H. Kiyata, H. Okushi, K. Okano, Y. Akiba, T. Kurosu, and M. Iida, Appl. Phys. Lett. 61, 1808 (1992).

${ }^{22}$ Y. Mokuno, A. Chayahara, Y. Soda, Y. Horino, and N. Fujimori, Diamond Relat. Mater. 14, 1743 (2005). 